Clínica Psiquiátrica Universitaria. Hospital Clínico. Facultad de Medicina. Universidad de Chile. Santiago, Chile. ¿Escuela de Psicología. Universidad de los Andes. Santiago, Chile

${ }^{3}$ Centro de Medicina Reproductiva y Desarrollo Integral del Adolescente,

Facultad de Medicina (CEMERA), Universidad de Chile.

${ }^{4}$ Hospital Regional de Rancagua. Rancagua, Chile. ${ }^{5}$ Facultad de Medicina. Universidad de los Andes. Santiago, Chile

UUnidad Académica de Psiquiatría. Universidad de Bristol. Bristol, Gran Bretaña.

aAlumna del Programa Conjunto del Doctorado en Salud Pública y Especialidad en Psiquiatría Adultos,

Escuela de Postgrado

Universidad de Chile.

Fuente de Financiamiento del estudio: Wellcome Trust (project number 082584 $\mathrm{Z} / 07 / \mathrm{Z})$

Recibido el 3 de junio de 2011, aceptado el 3 de noviembre de 2011

Correspondencia a:

Dra. Viviana Guajardo Universidad de Chile Avenida La Paz 1003 Santiago. Fono: 9788601

E-mail:vguajardo@med. uchile.cl

\section{Consumo de sustancias y síntomas depresivos en escolares de liceos municipales de Santiago}

\author{
GRACIELA ROJAS ${ }^{1}$, JORGE GAETE ${ }^{2}$, VIVIANA GUAJARDO ${ }^{1, a}$, \\ VANIA MARTÍNEZ ${ }^{3}$, SERGIO BARROILHET ${ }^{2}$, JUAN MENESES ${ }^{4}$, \\ ROSEMARIE FRITSCH ${ }^{1,5}$, RICARDO ARAYA ${ }^{6}$
}

\section{Association between drug consumption and depression among high school students}

Background: The presence of emotional problems may trigger drug abuse. Aim: To determine the association between illicit drug use and severity of depression symptoms among students of public secondary schools in Santiago, Chile. Material and Methods: A probabilistic sample of 2,597 adolescents aged between 12 and 18 years ( $45 \%$ women), attending first year in public high-schools in Santiago, answered self-report questionnaires about consumption, during the last 30 days , of alcohol, tobacco and marihuana, and the Beck Depression Inventory - II. Logistic regression models were used to study the association between drug use and severity of depression symptoms, adjusting by sex. Results: Tobacco and marihuana use was reported by 38 and 13\% of students, respectively. Frequency of consumption was significantly higher among women. Mild, moderate or severe depressive symptoms were found among $16 \%, 14 \%$ and $9 \%$ of students, respectively. Depressive symptoms were more common among women. There was a significant association between drug use and severity of depression among participants of both genders. Conclusions: The association between depressive symptoms and drug use should be considered on the design of treatment guidelines for these health problems in Chile.

(Rev Med Chile 2012; 140: 184-191).

Key words: Adolescent; Alcohol; Depression; Street drugs. $\sim$ hile es un país que se ha caracterizado por contar con buenos datos epidemiológicos en salud mental, lo que le ha permitido planificar reconocidas políticas públicas ${ }^{1-3}$.

El Consejo Nacional para el Control de Estupefacientes (CONACE) que actualmente corresponde al Servicio Nacional para la Prevención y Rehabilitación del Consumo de Drogas y Alcohol (SENDA) ha realizado estudios de consumo de sustancias en la población escolar chilena desde el año $1995^{4}$; y participado con las Comisiones de Drogas de Argentina, Bolivia, Ecuador, Perú y Uruguay, y con la Oficina de las Naciones Unidas contra la Droga y el Delito y la Comisión Interamericana para el Control del Abuso de Drogas de la Secretaría de Seguridad Multidimensional de la Organización de los Estados Americanos. En el año 2007 se realizó el segundo estudio conjunto en población escolarizada entre 13 y 17 años que incluyó una muestra de 170.286 escolares de 6 países. Según este estudio, Chile se encuentra en el segundo lugar en la prevalencia mensual de consumo de alcohol $(53,19 \%)$, después de Argentina $(60,19 \%)$. En prevalencia mensual de consumo de tabaco, se ubica en el primer lugar (32,58\%). En el caso del alcohol, Chile es el único país de los estudiados que muestra un mayor consumo de alcohol en las mujeres. Otro aspecto preocupante que arrojó este estudio es que Chile tiene la prevalencia anual del consumo de marihuana más alta 
$(14,3 \%)$. En el caso de la marihuana, así como en otras sustancias ilícitas, el consumo anual es más frecuente en hombres que en mujeres ${ }^{5}$.

En el país se han llevado a cabo dos importantes estudios epidemiológicos de trastornos psiquiátricos en población general mayor de 15 años utilizando entrevistas estructuradas. Araya y cols encontraron una prevalencia actual de episodio depresivo de 5,5\% ${ }^{4-7}$ en una población de 16-64 años, usando criterios CIE- $10^{2}$. Mientras que Vicente y cols usando criterios DSM-III-R, informaron una prevalencia de episodio depresivo en los últimos 6 meses de 4,7\% (DE 0,4) ${ }^{6}$. En ambos estudios, los cuadros depresivos fueron más prevalentes en mujeres que en hombres.

En los últimos 20 años han aparecido estudios que dan cuenta de la prevalencia de síntomas depresivos en adolescentes, con una amplia variabilidad, dada por el uso de diferentes metodologías e instrumentos de evaluación ${ }^{7-12}$. La prevalencia de sintomatología depresiva clínicamente significativa, varió entre $13,2 \%{ }^{10}$ y $44 \%{ }^{11}$. Estos estudios han incluido muestras de adolescentes entre 12 y 20 años. En todos los estudios, las mujeres presentan una mayor prevalencia que los hombres.

La literatura internacional muestra una relación estrecha entre consumo de sustancias y síntomas depresivos entre adolescentes, aunque no hay consenso en la relación temporal entre ellos, tampoco sobre las diferencias de sexo, ni sobre los orígenes de esta asociación ${ }^{13-15}$.

Algunos estudios han encontrado que la depresión predice problemas con el consumo de alcohol ${ }^{16}$, y otros que el consumo problemático de alcohol u otras drogas predicen depresión ${ }^{17-21}$. También hay quienes concluyen que hay una relación recíproca entre ellos ${ }^{22-26}$.

Brook y cols (2002) encontraron que el consumo de alcohol, marihuana y otras drogas en la adolescencia, predice el desarrollo de un episodio depresivo mayor al llegar a la adultez ${ }^{27}$.

Por último, un estudio realizado por Marmorstein (2009) a una muestra comunitaria de 20.728 adolescentes estadounidenses, evidenció que la asociación entre problemas de consumo de alcohol y síntomas depresivos difería según la edad y el sexo, mostrando que altos niveles de síntomas depresivos se relacionan con mayores niveles iniciales de problemas de consumo alcohol particularmente en las mujeres, así como incrementos más rápidos en el tiempo de consumo problemático de alcohol en los hombres. Altos niveles de consumo problemático de alcohol se asociaron con mayores niveles iniciales de síntomas depresivos, especialmente en las mujeres ${ }^{26}$.

En Chile, no existen a la fecha datos sobre esta asociación en adolescentes.

El objetivo de esta comunicación es determinar la asociación entre consumo de alcohol, tabaco, marihuana y la intensidad de la sintomatología depresiva en una muestra de escolares de primero medio de liceos de Santiago de Chile.

\section{Material y Método}

Los datos aquí analizados corresponden al diagnóstico basal del estudio "Intervención Escolar para mejorar la Salud Mental de jóvenes de Enseñanza Media: Yo Pienso, Siento y Actúo", que es un ensayo clínico controlado y aleatorio que tuvo como objetivo medir la efectividad de una intervención universal para mejorar la salud mental de una muestra representativa de establecimientos educacionales municipalizados de Santiago.

\section{Participantes}

Se utilizó la base de datos del Ministerio de Educación ${ }^{31}$ como marco muestral. El universo estuvo constituido por 95 liceos. Fueron estratificados de acuerdo al estrato socioeconómico, utilizando el Índice de Vulnerabilidad del Establecimiento calculado a través del método del Sistema Nacional de Asignación con Equidad de la Junta Nacional de Auxilio Escolar y Becas ${ }^{32}$, y el tamaño del establecimiento escolar. La selección se hizo de tal forma que todos los estratos estuvieran igualmente representados. Se excluyeron los liceos rurales, de educación especial, de adultos o vespertinos. Se seleccionaron en forma aleatoria 23 establecimientos municipalizados de la Provincia de Santiago, que fueran mixtos y tuvieran al menos 2 cursos de $1^{\circ}$ Enseñanza Media.

Una vez reclutados los colegios participantes, se procedió a evaluar a los alumnos en la sala de clases a través de un cuestionario auto-administrado.

La participación en las evaluaciones fue voluntaria y previo consentimiento de los padres y asentimiento de los/as alumnos/as, documentos aprobados por el Comité de Ética del Hospital Clínico de la Universidad de Chile. 
Consumo de sustancias y síntomas depresivos en escolares chilenos - G. Rojas et al

\section{Instrumentos}

Los cuestionarios fueron administrados en la sala de clases por un equipo de 22 evaluadores entrenados entre Marzo y Abril del 2009.

Esta evaluación consistió de una encuesta autoaplicada que incluía el Inventario de Depresión de Beck II para medir síntomas depresivos y siete preguntas sobre consumo, en los últimos 30 días, de alcohol, tabaco y marihuana ${ }^{30,31}$. Existen traducciones del BDI-II al castellano. Una ha sido aplicada por Penley y cols, en Estados Unidos de Norteamérica con población latina ${ }^{32}$. Otra traducción y estudio de sus propiedades psicométricas ha sido realizada en España $^{36}$. Una adaptación lingüística del inventario realizada por Melipillán y cols $(2008)^{34}$ en Chile mostró ser adecuada para adolescentes chilenos y fue usada en esta investigación. El BDI-II también ha sido utilizado previamente en nuestro país en muestras no clínicas de adolescentes ${ }^{11}$. Según Beck et al. un puntaje de 14 y más es considerado compatible con un episodio depresivo, un puntaje entre 0 y 13 sintomatología depresiva mínima o sin depresión, un puntaje entre 14 y 19 sintomatología depresiva leve, entre 20 y 28 puntos sintomatología depresiva moderada y un puntaje mayor a 29 sintomatología depresiva grave ${ }^{35}$.

\section{Análisis estadístico}

Se utilizó el paquete estadístico STATA 11.0. Se utilizaron descriptivos generales para las prevalencias de sintomatología depresiva y consumo de sustancias para hombres y mujeres. Se realizaron análisis de regresión logística para la asociación entre uso de tabaco, alcohol y marihuana y sintomatología depresiva, un análisis univariado para estudiar la asociación entre sintomatología depresiva y el consumo de sustancias en el último mes, modelos de regresión logística ajustados por edad y sexo y análisis para evaluar posibles interacciones entre sexo y consumo de sustancias moderando la presencia de sintomatología depresiva.

\section{Resultados}

\section{Descripción de la muestra}

La muestra estuvo constituida por 2597 escolares (44,6\% mujeres y 55,4\% hombres) de 23 establecimientos municipales y 69 cursos de Primero Medio. Al momento de la encuesta, los entrevistados tenían una edad promedio de 14,52 años (IC 95\%, 14,48-14,55) con un rango entre 12 y 18 años. Los establecimientos se dividieron en: aquellos que tenían 2 cursos $(\mathrm{N}=8)$, tres cursos $(\mathrm{N}=7)$ y cuatro o más cursos $(\mathrm{N}=8)$.

\section{Prevalencia en el último mes del consumo de alcohol, tabaco o marihuana}

La prevalencia de consumo de tabaco, alcohol o marihuana, en los últimos 30 días ascendía a $47,2 \%$, mayor en el sexo femenino. La diferencia entre los sexos fue estadísticamente significativa $\left(\chi^{2}=11,528 ; p=0,000\right)$.

La prevalencia de consumo de tabaco en los últimos 30 días ascendía a $38,3 \%$, siendo más prevalente en mujeres que en hombres $\left(\chi^{2}=22,284\right.$; $\mathrm{p}=0,000)$.

La prevalencia de consumo de alcohol en los últimos 30 días ascendía a 27,3\%, sin diferencias por sexo .

La prevalencia de consumo de marihuana en los últimos 30 días ascendía a 13,4\%; siendo más prevalente en mujeres que en hombres $\left(\chi^{2}=4,724\right.$; $\mathrm{p}=0,017)($ Tabla 1$)$.

\section{Prevalencia de sintomatología depresiva}

El $60,2 \%$ de la muestra (hombres 71,9\%; mujeres $45,5 \%$ ) no presentaba sintomatología depresiva; $16,4 \%$ presentó sintomatología depresiva leve (hombres 13,4\%; mujeres 20,1\%), 14,0\% moderada (hombres 9,9\%; mujeres 19, 1\%) y 9,4\% severa (hombres 4,7\%; mujeres 15,3\%) (Tabla 2).

\section{Asociación entre sintomatología depresiva y consumo de alcohol, tabaco y/o marihuana}

Al considerar si las poblaciones de adolescentes sin depresión (BDI-II < 14) y con depresión (BDIII $\geq 14$ ) se diferenciaban según el consumo mensual de sustancias, se encontró que el odds ratio (OR) no ajustado de tener depresión si consumió tabaco fue de 2,23 (IC 95\%, 1,82-2,73); si consumió alcohol fue de 1,87 (IC 95\%, 1,52-2,31), y si consumió marihuana fue de 2,05 (IC 95\%, 1,57$2,69)$. Cuando se ajustó por edad y sexo, los ORs respectivos fueron: 1,93 (IC 95\%, 1,59-2,34), 1,76 (IC 95\%, 1,41-2,18), y 1,73 (IC 95\%, 1,33-2,24).

$\mathrm{Al}$ estudiar la intensidad del consumo y la presencia o no de síntomas depresivos, se encontró que, en general, a mayor consumo de tabaco, alcohol o marihuana, mayor probabilidad de tener un BDI-II $\geq 14$, aun después de ajustar por edad y sexo (Tabla 3 ).

El estudio de la asociación entre intensidad 
Tabla 1. Consumo de sustancias últimos 30 días según sexo

\begin{tabular}{|lcccccccc|}
\hline & \multicolumn{2}{c}{ Total } & \multicolumn{2}{c}{ Hombres } & \multicolumn{2}{c}{ Mujeres } & $\boldsymbol{\chi}^{\mathbf{2}}$ & (p value) \\
& $\mathbf{n}$ & $\mathbf{\%}$ & $\mathbf{n}$ & $\mathbf{\%}$ & $\mathbf{n}$ & $\mathbf{\%}$ & & \\
\hline Consumo tabaco & 993 & 38,3 & 493 & 34,3 & 500 & 43,3 & 22,284 & $(0,000)$ \\
\hline Consumo alcohol & 706 & 27,3 & 387 & 27,0 & 319 & 27,6 & 0,112 & $(0,386)$ \\
\hline Consumo marihuana & 348 & 13,4 & 174 & 12,1 & 174 & 15,1 & 4,724 & $(0,017)$ \\
\hline Cualquier consumo & 1.220 & 47,2 & 633 & 44,2 & 587 & 50,9 & 11,528 & $(0,000)$ \\
\hline
\end{tabular}

Tabla 2. Intensidad sintomatología depresiva según sexo

\begin{tabular}{|lcccccccc|}
\hline Sintomatología & \multicolumn{2}{c}{ Total } & \multicolumn{2}{c}{ Hombres } & \multicolumn{2}{c|}{ Mujeres } & Z value* & (p value) \\
& $\mathbf{n}$ & $\mathbf{\%}$ & $\mathbf{n}$ & $\mathbf{\%}$ & $\mathbf{n}$ & $\%$ & & \\
Mínima o sin depresión (0-13) & 1.563 & 60,2 & 1.036 & 71,9 & 527 & 60,2 & 13,96 & 0,000 \\
Leve (14-19) & 425 & 16,4 & 193 & 13,4 & 232 & 20,1 & $-4,82$ & 0,000 \\
Moderada (20-28) & 364 & 14,0 & 143 & 9,9 & 221 & 19,1 & $-3,87$ & 0,000 \\
\hline Severa (29 o más) & 245 & 9,4 & 68 & 4,7 & 177 & 15,3 & $-8,66$ & 0,000 \\
\hline Total & 2.597 & 100 & 1.440 & 100 & 1.157 & 100 & & \\
\hline
\end{tabular}

*Pr test: Test de Proporciones de 2 muestras.

Tabla 3. Riesgo de depresión (BDI-II $\geq 14$ ) según intensidad del consumo de sustancias

\begin{tabular}{|c|c|c|c|}
\hline & & $\begin{array}{l}\text { No ajustado } \\
\text { OR (IC } 95 \%)\end{array}$ & $\begin{array}{l}\text { Ajustado* } \\
\text { OR (IC 95\%) }\end{array}$ \\
\hline \multirow{5}{*}{$\begin{array}{l}\text { Tabaco: Número de cigarrillos consumidos } \\
\text { en promedio al día en último mes }\end{array}$} & Menos de 1 al día (referencia) & 1 & 1 \\
\hline & $1-5$ & $1,87(1,50-2,33)$ & $1,58(1,30-1,93)$ \\
\hline & 6-10 & $2,52(1,74-3,66)$ & $2,12(1,29-3,46)$ \\
\hline & $11-20$ & $1,96(1,18-3,27)$ & $1,66(0,80-3,46)$ \\
\hline & Más de 20 & $3,10(2,14-4,49)$ & $2,66(1,83-3,86)$ \\
\hline \multirow{5}{*}{$\begin{array}{l}\text { Alcohol: Número de tragos consumidos en } \\
\text { promedio al día en último mes }\end{array}$} & No ha bebido (referencia) & 1 & 1 \\
\hline & 1 ó 2 & $1,46(1,12-1,90)$ & $1,38(1,06-1,80)$ \\
\hline & 3 ó 4 & $2,40(1,75-3,28)$ & $2,10(1,49-2,97)$ \\
\hline & 5 ó 6 & $2,10(1,26-3,48)$ & $2,01(1,11-3,65)$ \\
\hline & 7 ó más & $3,39(1,70-6,77)$ & $3,41(1,70-6,83)$ \\
\hline \multirow{6}{*}{$\begin{array}{l}\text { Marihuana: Número de veces que ha } \\
\text { consumido marihuana en últimos } 30 \text { días }\end{array}$} & Nunca (referencia) & 1 & 1 \\
\hline & 1 ó 2 & $1,84(1,27-2,69)$ & $1,58(1,08-2,32)$ \\
\hline & 3 ó 5 & $2,20(1,34-3,61)$ & $1,98(1,20-3,27)$ \\
\hline & 6 ó 9 & $4,63(2,07-10,35)$ & $3,26(1,37-7,77)$ \\
\hline & 10 ó 19 & $2,14(0,84-5,48)$ & $1,51(0,57-3,99)$ \\
\hline & Más de 20 & $1,72(0,94-3,16)$ & $1,50(0,80-2,82)$ \\
\hline
\end{tabular}

Nota: *Regresión logística, usando como variables de control sexo y edad. 
Consumo de sustancias y síntomas depresivos en escolares chilenos - G. Rojas et al

Tabla 4. Asociación* entre sintomatología depresiva y consumo de alcohol, tabaco y/o marihuana**

\begin{tabular}{|c|c|c|c|c|c|c|c|c|}
\hline \multirow[t]{2}{*}{ Variable } & \multicolumn{2}{|c|}{$\begin{array}{l}\text { Consumo } \\
\text { cualquiera }\end{array}$} & \multicolumn{2}{|c|}{$\begin{array}{c}\text { Consumo } \\
\text { alcohol }\end{array}$} & \multicolumn{2}{|c|}{$\begin{array}{c}\text { Consumo } \\
\text { tabaco }\end{array}$} & \multicolumn{2}{|c|}{$\begin{array}{l}\text { Consumo } \\
\text { marihuana }\end{array}$} \\
\hline & $\%$ & $\begin{array}{c}\text { OR } \\
\text { (IC 95\%) }\end{array}$ & $\%$ & $\begin{array}{c}\text { OR } \\
\text { (IC 95\%) }\end{array}$ & $\%$ & $\begin{array}{c}\text { OR } \\
\text { (IC 95\%) }\end{array}$ & $\%$ & $\begin{array}{c}\text { OR } \\
\text { (IC 95\%) }\end{array}$ \\
\hline \multicolumn{9}{|l|}{ Sexo } \\
\hline Hombres & 44,2 & 1 & 27,0 & 1 & 34,3 & 1 & 12,1 & 1 \\
\hline Mujeres & 50,9 & $\begin{array}{c}1,04 \\
(0,88-1,22)\end{array}$ & 27,6 & $\begin{array}{c}0,84 \\
(0,66-1,06)\end{array}$ & 43,3 & $\begin{array}{c}1,18 \\
(1,03-1,36)\end{array}$ & 15,1 & $\begin{array}{c}1,02 \\
(0,78-1,34)\end{array}$ \\
\hline \multicolumn{9}{|c|}{ Sintomatología depresiva } \\
\hline Mínima & 39,0 & 1 & 22,2 & 1 & 30,8 & 1 & 10,0 & 1 \\
\hline Leve & 56,4 & $\begin{array}{c}2,01 \\
(1,59-2,53)\end{array}$ & 32,7 & $\begin{array}{c}1,76 \\
(1,38-2,26)\end{array}$ & 45,8 & $\begin{array}{c}1,84 \\
(1,47-2,30)\end{array}$ & 15,8 & $\begin{array}{c}1,68 \\
(1,28-2,20)\end{array}$ \\
\hline Moderada & 58,1 & $\begin{array}{c}2,15 \\
(1,70-2,73)\end{array}$ & 33,3 & $\begin{array}{c}1,83 \\
(1,36-2,47)\end{array}$ & 48,8 & $\begin{array}{c}2,05 \\
(1,57-2,68)\end{array}$ & 17,1 & $\begin{array}{c}1,84 \\
(1,27-2,65)\end{array}$ \\
\hline Severa & 67,4 & $\begin{array}{c}3,19 \\
(2,24-4,55)\end{array}$ & 40,8 & $\begin{array}{c}2,58 \\
(1,88-3,55)\end{array}$ & 58,0 & $\begin{array}{c}2,91 \\
(2,04-4,16)\end{array}$ & 25,7 & $\begin{array}{c}3,08 \\
(2,21-4,31)\end{array}$ \\
\hline
\end{tabular}

Nota: *Regresión Logística. Variables explicativas: sexo e intensidad de sintomatología. **El consumo de cada sustancia fue definido como el haber consumido alcohol, tabaco o marihuana durante el último mes.

de la sintomatología depresiva y el consumo de alcohol, marihuana y/o tabaco ajustada por edad y sexo reveló que, a mayor intensidad depresiva, mayor es la probabilidad de consumir tabaco, alcohol, o marihuana en el último mes en ambos sexos (Tabla 4). No se encontraron interacciones entre el sexo y la intensidad depresiva.

\section{Discusión}

Nuestros resultados confirman la asociación entre síntomas depresivos y consumo de sustancias descrita internacionalmente ${ }^{36}$. Se encontró que a mayor cantidad del consumo de una sustancia, sea tabaco, alcohol o marihuana, mayor es la probabilidad de que adolescentes presenten síntomas depresivos clínicamente significativos; y la asociación se hace más marcada a mayor intensidad de la sintomatología depresiva. Varias hipótesis se han propuesto para esta asociación, especialmente aquella que sostiene que las sustancias serían consumidas como automedicación para calmar la sintomatología depresiva ${ }^{26,37,38}$. El grupo de investigación no tiene data para poder explorar las motivaciones que tuvieron los adolescentes para consumir sustancias dado que tenían síntomas depresivos, y debido a que este es un análisis transversal, no podemos establecer una asociación causal debido al efecto de causalidad reversa $^{39}$. Siguiendo este último argumento, la asociación pudiera ser inversa, y se podría hipotetizar que el consumo de estas sustancias produjeron cambios en el funcionamiento psíquico de estos adolescentes. Varios estudios longitudinales han establecido la asociación temporal entre el consumo de tabaco ${ }^{40}$, alcohol ${ }^{26}$ y marihuana ${ }^{41}$ y el desarrollo de síntomas depresivos. Si bien esta es una asociación conocida, este es el primer estudio en Chile que demuestra esta asociación en adolescentes. Sólo estudios longitudinales nos podrán ayudar a conocer mejor la relación temporal entre depresión y consumo de sustancias.

Nuestro estudio mostró que 39,8\% de los escolares encuestados tuvieron clínicamente síntomas depresivos (puntaje en el BDI-II $\geq 13$ ) y que las mujeres tuvieron mayor prevalencia e intensidad de la sintomatología que los hombres. Las cifras parecen ser consistentes con otros estudios a nivel nacional ${ }^{10-12}$, y superiores a las comunicadas por la literatura internacional ${ }^{35,36}$. La diferencia entre los sexos es un hecho ya conocido de la epidemiología psiquiátrica $^{37}$.

Nuestros hallazgos indican que en el trata- 
miento de los adolescentes deprimidos debe ser considerado el estudio del uso de sustancias de abuso y viceversa, debido a la alta asociación entre ambas variables. Sin embargo, las actuales políticas y guías de atención de salud de estas patologías, no consideran esta asociación y no han incorporado evaluaciones rutinarias que permitiesen establecer la asociación mutua. A este hecho hay que agregar que la garantía de acceso a tratamiento de las personas deprimidas sólo rige a partir de los 15 años de edad.

Por otro lado, este estudio muestra que tabaco y alcohol son las sustancias más consumidas en el último mes. Estos resultados son coincidentes con los encontrados a nivel nacional y latinoamericano. Otro de los hallazgos que se replica es que, la prevalencia del último mes del consumo de tabaco es mayor entre las mujeres que en los hombres. Sin embargo, una diferencia detectada con los estudios de CONACE, es que desde el 2003, la sustancia más consumida durante el último mes por la población escolar nacional es el alcohol, mientras que en nuestro estudio fue el tabaco. El consumo de alcohol en nuestro estudio (27,3\%) es menor a la informada para la población escolar chilena el $2009(36 \%)^{34}$. Una posible explicación a esta diferencia es que nuestro estudio incluyó sólo estudiantes de colegios municipalizados y consistentemente se ha encontrado que el consumo de alcohol es menor en colegios municipalizados que en colegios particulares o subvencionados ${ }^{34}$. En esta diferencia la disponibilidad de dinero para acceder al consumo puede jugar un rol explicativo.

Por otro lado, en el último estudio comparativo de las prevalencias del consumo de sustancias en escolares latinoamericanos ${ }^{2}$; la prevalencia en Chile del consumo de alcohol fue de 53,19\% (hombres, 53,12\%; mujeres, 53,31\%). Nuestro estudio ratifica que el consumo de alcohol entre mujeres es ligeramente superior al de los hombres ${ }^{2}$.

Adicionalmente, una de las diferencias más llamativas es la alta prevalencia en el último mes del consumo de marihuana en los escolares de nuestro estudio, siendo mayor en mujeres $(15,1 \%)$ que en hombres $(12,1 \%)$. El último estudio de CONACE muestra que la prevalencia en el último mes fue de $8 \%$ en la población escolar general. No hay datos disponibles aún, desde los estudios de CONACE que nos muestren la diferenciación por tipo de colegio del consumo de marihuana. Sin embargo, es plausible que el grupo de colegios municipalizados seleccionados en nuestro estudio, esté dando cuenta de una población escolar más vulnerable, lo que puede explicarse por el éxodo de estudiantes de la educación municipal.

\section{Fortalezas y limitaciones}

Se trata de una muestra importante, representativa de escolares de primero medio en colegios municipalizados de Santiago. Se utilizaron instrumentos conocidos para las variables que se midieron.

Sin embargo, se trata de una muestra que fue calculada para un ensayo clínico y no para un estudio de prevalencia por lo cual las conclusiones pueden ser limitadas. Por otro lado, la muestra incluyó sólo a estudiantes de colegios municipalizados, es decir, en los que factores sociales asociados a la pobreza pudieran estar ejerciendo una influencia importante para la expresión de los síntomas y conductas medidas. Esto hace que los resultados no puedan ser generalizables al resto de la población escolar chilena.

Los resultados del ensayo clínico que se está llevando a cabo y que medirá la eficacia de una intervención grupal para mejorar la salud mental de estos jóvenes nos informará sobre el impacto que pueda tener influir sobre una u otra variable.

Agradecimientos: A los alumnos que han entregado esta información. A los profesores y evaluadores que han hecho posible la recolección de esta información.

\section{Referencias}

1. Vicente B, Kohn R, Rioseco P, Saldivia S, Levav I, Torres S. Lifetime and 12-month prevalence of DSM-III-R disorders in the Chile psychiatric prevalence study. Am J Psychiatry 2006; 163 (8): 1362-70.

2. Araya R, Rojas G, Fritsch R, Acuña J, Lewis G. Common mental disorders in Santiago, Chile: Prevalence and socio-demographic correlates. The British Journal of Psychiatry 2001; 178 (3): 228-33.

3. Araya R, Rojas G, Fritsch R, Gaete J, Rojas M, Simon G, et al. Treating depression in primary care in low-income women in Santiago, Chile: a randomised controlled trial. Lancet 2003; 361 (9362): 995-1000.

4. Gobierno de Chile MdISdIC. Séptimo Estudio Nacional de Drogas en la Población Escolar de Chile, 2007. Santiago, Chile: Ministerio de Interior, Chile; 2008.

5. Oficina de Naciones Unidas Contra las Drogas y el De- 
Consumo de sustancias y síntomas depresivos en escolares chilenos - G. Rojas et al

lito. Segundo Estudio Conjunto sobre el Consumo de Drogas en población escolarizada de Nivel Medio. 2010.

6. Vicente B, Kohn R, Rioseco P, Saldivia S, Baker C, Torres S. Population prevalence of psychiatric disorders in Chile: 6-month and 1-month rates. The British Journal of Psychiatry 2004; 184 (4): 299-305.

7. Sepúlveda G, Almonte C, Valenzuela C, Avendaño A. Estilos de socialización de los padres y desarrollo psicosocial en adolescentes de 16 a 19 años / Parental socialization styles and psychosocial development in adolescents. Revista Chilena de Pediatría 1991; 62 (6): 396-403.

8. De la Barra F, Toledo V y Rodríguez J. Estudio de Salud mental en niños escolares de Santiago Occidente IV: trastornos psiquiátricos, diagnóstico social y discapacidad. Rev Chil Neuropsiquiat 2004, 42: 4, 250-72.

9. Vicente B, Saldivia S, Rioseco P, de la Barra F, Valdivia M, Melipillan R, et al. Epidemiología de trastornos mentales en la provincia de Cautín. Rev Med Chile 2010; 138: 965-74.

10. Cumsille P, Martínez ML. Síntomas de depresión en estudiantes de enseñanza media de Santiago. Revista Chilena de Pediatria 1997; 68 (2): 74-7.

11. Gellona J, Zarraonandia A, Muñoz R, Flores M. Prevalencia de síntomas depresivos en escolares adolescentes de dos colegios del sector oriente de Santiago/Depressisve symptoms prevalence in adolescent students of two Santiago schools. Psiquiatra y Salud Mental 2005; 22 (1/2): 93-9.

12. Cova F, Melipillan R, Valdivia M, Bravo E, Valenzuela B. Sintomatología depresiva y ansiosa en estudiantes de enseñanza media. Revista Chilena de Pediatria 2007; 78 (2): 151-9.

13. Hallfors DD, Waller MW, Bauer D, Ford CA, Halpern CT. Which comes first in adolescence-sex and drugs or depression? Am J Prev Med 2005; 29 (3): 163-70.

14. Rao U. Links between depression and substance abuse in adolescents: neurobiological mechanisms. Am J Prev Med 2006; 31 (6 Suppl 1): S161-74.

15. Henry B, Feehan M, McGee R, Stanton W, Moffitt TE, Silva P. The importance of conduct problems and depressive symptoms in predicting adolescent substance use. J Abnorm Child Psychol 1993; 21 (5): 469-80.

16. Abraham HD, Fava M. Order of onset of substance abuse and depression in a sample of depressed outpatients. Compr Psychiatry 1999; 40 (1): 44-50.

17. Bovasso GB. Cannabis abuse as a risk factor for depressive symptoms. Am J Psychiatry 2001; 158 (12): 2033-7.

18. Bukstein OG, Glancy LJ, Kaminer Y. Patterns of affective comorbidity in a clinical population of dually diagnosed adolescent substance abusers. J Am Acad Child Adolesc Psychiatry 1992; 31 (6): 1041-5.
19. Rao U, Daley SE, Hammen C. Relationship between depression and substance use disorders in adolescent women during the transition to adulthood. J Am Acad Child Adolesc Psychiatry 2000; 39 (2): 215-22.

20. Rohde P, Lewinsohn PM, Kahler CW, Seeley JR, Brown RA. Natural course of alcohol use disorders from adolescence to young adulthood. J Am Acad Child Adolesc Psychiatry 2001; 40 (1): 83-90.

21. Stice E, Burton EM, Shaw H. Prospective relations between bulimic pathology, depression, and substance abuse: unpacking comorbidity in adolescent girls. J Consult Clin Psychol 2004; 72 (1): 62-71.

22. Clark DB, Pollock N, Bukstein OG, Mezzich AC, Bromberger JT, Donovan JE. Gender and comorbid psychopathology in adolescents with alcohol dependence. J Am Acad Child Adolesc Psychiatry 1997; 36 (9): 1195-203.

23. Deykin EY, Buka SL, Zeena TH. Depressive illness among chemically dependent adolescents. Am J Psychiatry 1992; 149 (10): 1341-7.

24. Hettema JM, Prescott CA, Kendler KS. The effects of anxiety, substance use and conduct disorders on risk of major depressive disorder. Psychol Med 2003; 33 (8): 1423-32.

25. Swendsen JD, Merikangas KR, Canino GJ, Kessler RC, Rubio-Stipec M, Angst J. The comorbidity of alcoholism with anxiety and depressive disorders in four geographic communities. Compr Psychiatry 1998; 39 (4): 176-84.

26. Marmorstein NR. Longitudinal associations between alcohol problems and depressive symptoms: early adolescence through early adulthood. Alcohol Clin Exp Res 2009; 33 (1): 49-59.

27. Brook DW, Brook JS, Zhang C, Cohen P, Whiteman M. Drug use and the risk of major depressive disorder, alcohol dependence, and substance use disorders. Arch Gen Psychiatry 2002; 59 (11): 1039-44.

28. Departamento de Estudios y Desarrollo. MINEDUC. Bases de datos oficiales del Ministerio de Educación. Estudios y estadísticas del sistema escolar chileno. Estadísticas e Indicadores. 2010.

29. Junta Nacional de Auxilio Escolar y Becas (JUNAEB). Indice de Vulnerabilidad del establecimiento (IVE)Sistema Nacional de Asignación con Equidad (SINAE). 2010.

30. Dozois DJA, Dobson KS, Ahnberg JL. A psychometric evaluation of the Beck Depression Inventory-II. Psychological Assessment 1988; 10: 83-9.

31. Krefetz DG, Steer RA, Gulab NA, Beck AT. Convergent validity of the Beck depression inventory-II with the reynolds adolescent depression scale in psychiatric inpatients. J Pers Assess 2002; 78 (3): 451-60.

32. Penley JA, Wiebe JS, Nwosu A. Psychometric properties 
of the Spanish Beck Depression Inventory-II in a medical sample. Psychol Assess 2003; 15 (4): 569-77.

33. Sanz J, Navarro ME, Vasquez C. Adaptación española del inventario para la depresión de Beck-II (BDI-II): 1. Propiedades psicométricas en estudiantes universitarios. Anal Modif Conduc 2003; 29: 239-88.

34. Melipillan R, Cova F, Rincon P, Valdivia M. Propiedades Psicométricas del Inventario de Depresión de Beck-II en Adolescentes Chilenos. Terapia Psicológica 2008; 26 (1): 59-69.

35. Beck AT, Steer RA, Brown GK. BDI-II. Beck depression Inventory-Second Edition. Manual. San Antonio, TX: The Psychological Coorporation; 1996.

36. Boys A, Farrell M, Taylor C, Goodman R, Brugha T, Bebbington $\mathrm{P}$, et al. Psychiatric morbidity and substance use in young people aged 13-15 years: results from the Child and Adolescent Survey of Mental Health. Bristish Journal of Psychiatry 2003; 182: 509-17.
37. Ferigolo M, Stein AT, Fuchs FD, Barros HM. Influence of depression and early adverse experiences on illicit drug dependence: a case-control study. Rev Bras Psiquiatr 2009; 31 (2): 106-13.

38. Libby AM, Orton HD, Stover SK, Riggs PD. What came first, major depression or substance use disorder? Clinical characteristics and substance use comparing teens in a treatment cohort. Addict Behav 2005; 30 (9): 1649-62.

39. Rothman KJ, Greenland S, Lash TL. Modern Epidemio$\log y$. Third ed. Philadelphia, PA: Lippincott Williams \& Wilkins; 2008.

40. Martini S, Wagner FA, Anthony JC. The association of tobacco smoking and depression in adolescence: evidence from the United States. Subst Use Misuse 2002; 37 (14): 1853-67.

41. Patton GC, Coffey C, Carlin JB, Degenhardt L, Lynskey M, Hall W. Cannabis use and mental health in young people: cohort study. BMJ 2002; 325 (7374): 1195-8. 